\title{
Experimental investigation of the longitudinal beam dynamics in a photoinjector using a two-macroparticle bunch
}

\author{
P. Piot, ${ }^{1,2, *}$ R. Tikhoplav, ${ }^{3, \dagger}$ D. Mihalcea, ${ }^{1}$ and N. Barov ${ }^{1, *}$ \\ ${ }^{1}$ Northern Illinois University, Department of Physics, DeKalb, Illinois 60115, USA \\ ${ }^{2}$ Fermi National Accelerator Laboratory, P.O. Box 500, Batavia, Illinois 60510, USA \\ ${ }^{3}$ University of Rochester, Department of Physics \& Astronomy, Rochester, New York 14627, USA \\ (Received 21 March 2006; published 18 May 2006)
}

We have developed a two-macroparticle bunch to explore the longitudinal beam dynamics through various components of the Fermilab/NICADD photoinjector. Such a two-macroparticle bunch is generated by splitting the ultraviolet pulse from the photocathode drive laser. The presented method allows the exploration of radio-frequency-induced compression in the 1.625 cell radio frequency gun and the booster cavity. It also allows a direct measurement of the momentum compaction of the magnetic bunch compressor. The measurements are compared with analytical and numerical models.

DOI: 10.1103/PhysRevSTAB.9.053501

PACS numbers: 29.27.- a, 41.85.-p, 41.75.Fr

\section{INTRODUCTION}

Linear accelerators designed to drive light sources based on the free-electron laser scheme [1] or advanced accelerator physics research and development experiments (e.g. plasma wake field accelerators [2]) need to provide electron bunches with small emittance and high peak current. In order to achieve such high-brightness beams, the bunch, after generation, is generally manipulated both in the transverse (e.g. emittance compensation in photoinjectors) and longitudinal (e.g. bunch compression) phase spaces. The beam dynamics associated with such beams is intricate since both external and internal fields play an equal role. It is, therefore, difficult to set up and optimize the beam manipulation process by simply measuring the bunch properties (i.e. emittances, momentum spread, bunch length, etc.). Instead, it is first necessary to make sure the lattice is set in a proper way, e.g., as devised by numerical simulations. Directly measuring the lattice properties is generally an easy task in the transverse phase space: one can simply perturb the beam trajectory with a magnetic steerer and study the perturbed orbit using beam position monitors. However, it is not such an easy matter as far as the longitudinal phase space is concerned. A method described in Refs. [3-6] uses a special cavity to measure the relative time of arrival of the bunch given a time or energy perturbation impressed upstream of the cavity. In the present paper we describe the implementation of a simpler method based on the generation of a "two-macroparticle bunch." Given the initial separation between the two macroparticles that compose the bunch, the longitudinal lattice properties can be inferred by studying the evolution of

\footnotetext{
*Electronic address: piot@nicadd.niu.edu

${ }^{\dagger}$ Current address: University of California, Los Angeles, California 90095, USA.

Current address: FAR-TECH, Inc., San Diego, California 92121, USA.
}

the macroparticle separation. The technique provides relevant information on the lattice properties provided the macroparticle charge is chosen to mitigate the possible interaction, e.g., due to space charge, between the two macroparticles. Possible other applications of such a technique include the characterization of bunch length diagnostics [7], and the investigation of collective effects such as long-range wake fields. In this latter, a nominally charged macroparticle is followed (or preceded) by a weakly charged macroparticle. The latter macroparticle "witnesses" the electromagnetic field associated to the former macroparticle.

This paper deals with the implementation of such a twomacroparticle bunch technique in the Fermilab/NICADD Photoinjector Laboratory (FNPL) for characterizing the single-particle longitudinal dynamics associated with a radio frequency (rf) gun, a superconducting accelerating rf cavity and a magnetic bunch compressor.

After describing the experimental setup in Sec. II, we present results of the technique and their comparison with numerical simulation in Secs. III and IV.

\section{EXPERIMENTAL SETUP}

Experimental tests of the two-macroparticle method were performed at FNPL [8]. An overview of FNPL is shown in Fig. 1: the accelerator consists of an electron source (rf gun) and a superconducting TESLA cavity [9] (booster cavity). The electron source is based on a 1.625cell rf cavity operating on the $\mathrm{TM}_{010, \pi}$ mode. An ultraviolet (uv) laser pulse $(\lambda=263 \mathrm{~nm})$ impinges a high-quantumefficiency cesium-telluride photocathode located on the back plate of the rf gun. The thereby photoemitted high charge $(Q \leq 20 \mathrm{nC})$ electron bunch has an energy of $\sim 4 \mathrm{MeV}$ upon exit from the rf gun. The bunch is further accelerated by the booster cavity and its final maximum energy reaches $16 \mathrm{MeV}$. The main operating parameters are summarized in Table I. Downstream of the cavity, the 


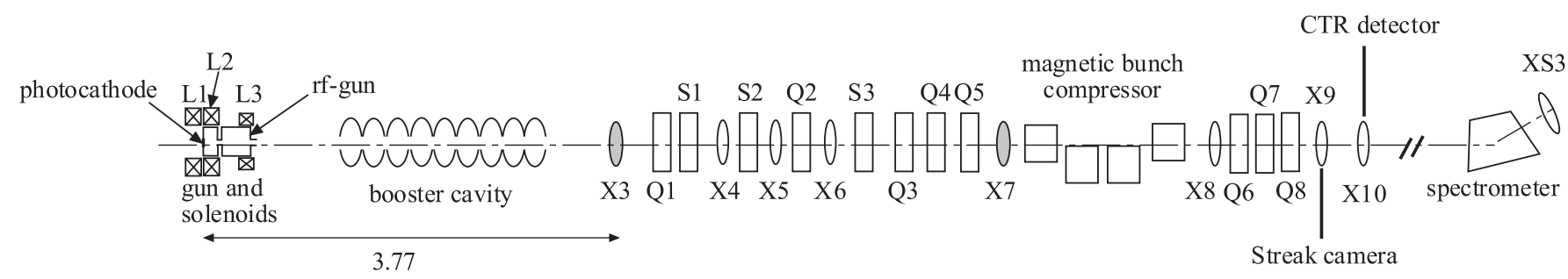

FIG. 1. Overview of the Fermilab/NICADD photoinjector. "X" refers to diagnostic stations (beam viewers and/or slit location), "L" to the solenoidal lenses, "Q" to quadrupoles, and "S" to skew quadrupoles. Distances are in meters. The magnetic bunch-compressor chicane bends the beam in the vertical plane while the spectrometer deflects the beam in the horizontal direction.

beam line includes a set of quadrupoles, steering dipoles, and a magnetic bunch-compressor chicane capable of enhancing the bunch peak current up to approximately $2.5 \mathrm{kA}$ [10]. The bunch compressor creates an orbit bump in the vertical plane. Downstream of the beam line, the electron beam can be horizontally bent by $45^{\circ}$ in a dispersive section equipped with a yttrium aluminum garnet screen (labeled XS3 in Fig. 1) for energy measurement. The horizontal dispersion value at XS3 location is $\left|\eta_{x}\right|=$ $317 \mathrm{~mm}$.

A time-domain bunch length measurement can be performed by a streak camera that streaks optical transition radiation pulses emitted as the bunch strikes the screen $\mathrm{X} 9$; see Fig. 1. The time resolution of the streak camera [11] when operated with the maximum sweep speed is approximately 2 ps (rms).

An alternative frequency-domain bunch length diagnostic based on Michelson interferometry of coherent transition radiation (CTR) is also available $[12,13]$. The bunch longitudinal density is monitored by detecting the CTR emitted as the electron bunch impinges screen X10. Under nominal operation of the diagnostics, the autocorrelation of the CTR pulse is recorded and the bunch length can then be indirectly estimated. For measurements reported hereafter, only the total power emitted via CTR emission was detected using a room-temperature optoacoustic detector [14]. In this mode of operation the diagnostics provide a

TABLE I. Typical settings for the rf-gun, accelerating section, and the photocathode drive laser. An accelerating phase of $90^{\circ}$ for the booster cavity corresponds to maximum energy gain.

\begin{tabular}{lcc}
\hline \hline \multicolumn{1}{c}{ Parameter } & Value & Units \\
\hline Laser injection phase & $45 \pm 5$ & degree \\
Laser pulse duration & $4( \pm 0.5)$ & $\mathrm{ps}$ \\
Bunch charge & $0-20$ & $\mathrm{nC}$ \\
$E_{z}$ on Cathode & $35 \pm 0.2$ & $\mathrm{MV} / \mathrm{m}$ \\
Booster-cavity accelerating gradient & $\sim 12$ & $\mathrm{MV} / \mathrm{m}$ \\
Booster-cavity accelerating phase & $\sim 84$ & degree \\
\hline \hline
\end{tabular}

way to minimize the bunch length (by maximizing the CTR emission).

The photoemission electron source offers a convenient way of producing a two-macroparticle bunch: the photocathode drive-laser pulse is split into two and then recombined in such a way that a time delay is introduced between the two pulses. The delay can be remotely varied from $\sim 7$ to $\sim 35 \mathrm{ps}$. A calibrated potentiometer provides a readout for the delay between the two pulses. An example of a double uv pulse is presented in Fig. 2. When such a doublepulse impinges the photocathode, it produces two electron bunches with a time separation much smaller than the $\mathrm{rf}$ period $\left(T_{\mathrm{rf}}=769 \mathrm{ps}\right)$. Hence both macroparticles fall into the same rf bucket and can be treated as a single bunch. Because of this latter feature, we henceforth refer to this
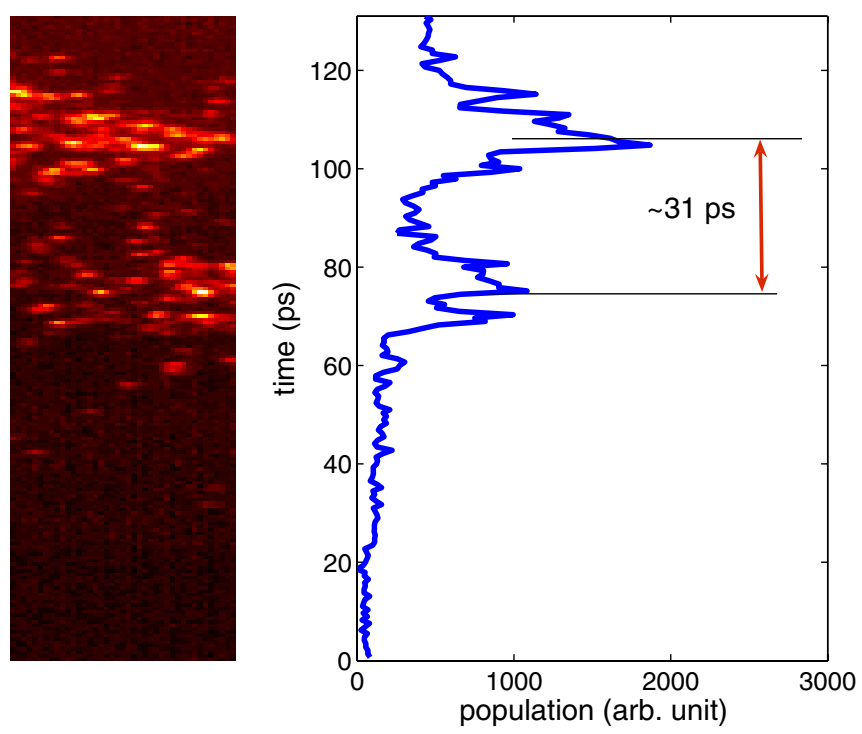

FIG. 2. (Color) Streak camera image (left) and corresponding time-profile (right) of a two-pulse laser. In this example the separation between the two pulses is approximately $31 \mathrm{ps}$. The apparent intensity difference between the two pulses (noticeable on the profiles) is an artifact of the measurement (slight misalignment of the second pulse on the streak camera entrance slit). 


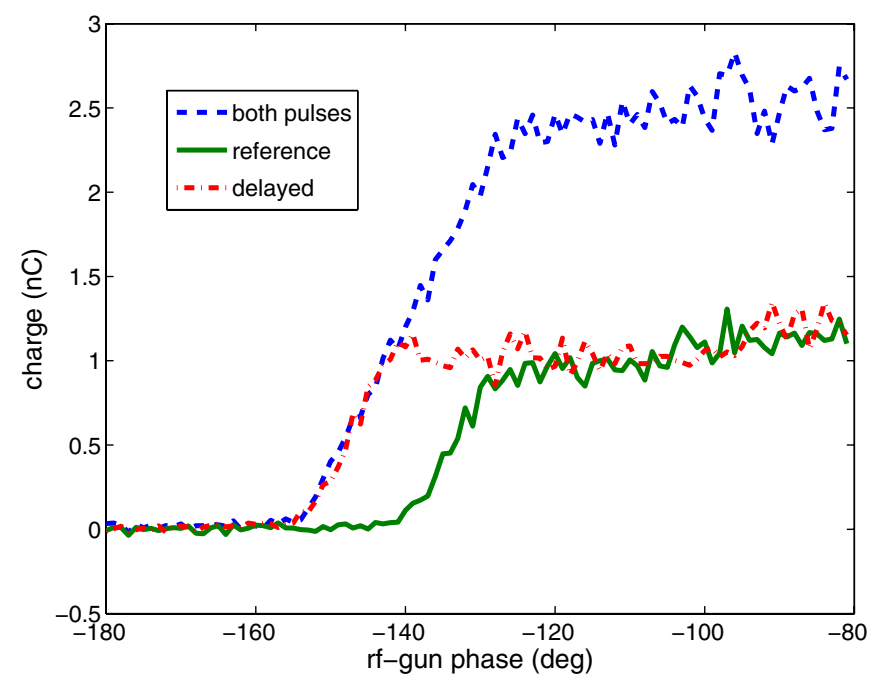

FIG. 3. (Color) Scan of the phase between the rf gun and the photocathode drive laser when only reference (delayed) or both uv pulses are incident on the photocathode. The data were obtained with the same uv-pulse configuration as in Fig. 2. The horizontal axis has an arbitrary offset.

two-electron-bunches system as a "two-macroparticle bunch." The macroparticle which is delayed is henceforth referred to as a delayed bunch (or "delayed" macroparticle) and the nondelayed one is called the "reference" bunch (or reference macroparticle). Either macroparticle can be "turned off" by blocking the associated uv-pulse. The delay between the two photoemitted bunches can be verified by scanning the phase between the electric field of the rf gun and the photocathode drive-laser clock. Such phase scans were recorded for various configurations of the two uv pulses (either of them or the two of them together). We present in Fig. 3 the results of such phase scans obtained for experimental conditions identical to the ones used to obtain the data of Fig. 2. From Fig. 3, we find that the phase shift between the emission of the two macroparticles is approximately $\simeq 15 \pm 2^{\circ}$ or $32 \pm 4 \mathrm{ps}$, which correlates well with the uv-pulse separation observed in Fig. 2.

\section{BUNCHING IN RF GUN AND BOOSTER CAVITY}

\section{A. Theoretical background}

In this section we briefly describe how the velocity bunching works. A detailed discussion is provided in Refs. [15-20]. An electron in a rf standing wave accelerating structure experiences a longitudinal electric field of the form

$$
E_{z}(z, t)=E_{0} \hat{E}_{z} \sin \left(\omega t+\psi_{o}\right),
$$

where $E_{0}$ is the peak field, $\hat{E}_{z}$ is the axial $E$-field distribution normalized to unity, $k \equiv 2 \pi /\left(c T_{\text {rf }}\right)$ the rf wave number, and $\psi_{0}$ the injection phase of the electron with respect to the rf wave. For the fundamental spatial distribution, $\hat{E}_{z}=\cos (k z)$. Let $\psi(z, t) \equiv \omega t-k z+\psi_{0}$ be the relative phase of the electron with respect to the wave. The evolution of $\psi(t, z)$ is

$$
\frac{d \psi}{d z}=\omega \frac{d t}{d z}-k=\frac{\omega}{\beta c}-k=k\left(\frac{\gamma}{\sqrt{\gamma^{2}-1}}-1\right),
$$

where $\gamma$ is the Lorentz factor. The energy gradient can be written as [16]

$$
\frac{d \gamma}{d z}=2 \alpha k \hat{E}_{z} \sin (\psi+k z)
$$

where $\alpha \equiv \frac{e E_{0}}{2 k m c^{2}}$ is the normalized accelerating field [16]. The system of coupled ordinary differential equations, (2) and (3), describes the longitudinal motion of an electron in the rf structure. There is no closed-form solution of such a system, and the equations of motion are generally numerically integrated.

References [16,17] treat the case of the rf gun. In [16] an approximate solution is derived using the method of successive approximation and a bunch-compression factor is inferred. However, both references deal with $1+n / 2$ cell cavities (where $n$ is an integer). At FNPL the half-cell of the $\mathrm{rf}$ gun is elongated leading to the presence of higher spatial harmonics. Therefore we numerically integrate the equation of motion using the field distribution $\hat{E}_{z}$ calculated with SUPERFISH [21].

The case of acceleration in the booster cavity is actually simpler: if we assume the cavity geometry only supports the fundamental space field distribution, then $\frac{d \gamma}{d z}=$ $\alpha k[\sin (\psi)+\sin (\psi+2 k z)]$. The backward wave (second term in the right-hand side of the latter equation) can be neglected and the system can be solved using the separation-of-variables technique to yield

$$
\alpha \cos \psi-\gamma+\sqrt{\gamma^{2}-1}=\mathcal{H}
$$

where $\mathcal{H}=\alpha \cos \psi_{0}-\gamma_{0}+\sqrt{\gamma_{0}^{2}-1}\left(\psi_{0}\right.$ and $\gamma_{0}$ being the initial injection phase and Lorentz factor). Equation (4) can be rewritten

$$
\psi(\gamma)=\arccos \left(\frac{\mathcal{H}+\gamma-\sqrt{\gamma^{2}-1}}{\alpha}\right),
$$

which upon differentiation yields the bunch-compression factor associated with the booster cavity:

$$
\mathcal{C}_{b} \equiv \frac{\partial \psi_{\infty}}{\partial \psi_{0}}=\frac{\sin \psi_{0}}{\sqrt{1-\left(\cos \psi_{0}-\frac{1}{2 \alpha \gamma_{0}}\right)^{2}}},
$$

where $\psi_{\infty} \equiv \lim _{\gamma \rightarrow \infty} \psi$. We assumed $\gamma_{0} \gg 1$ to derive the latter equation. For the booster cavity we have $\alpha \simeq 0.9$ and $\gamma_{0} \simeq 7$. 


\section{B. Experiment}

The nominal rf-gun phase is set to $45^{\circ}$ with respect to the zero-crossing phase, i.e., the phase value corresponding to a zero electric field on the photocathode. The boostercavity phase was tuned for maximum energy gain. The separation between the two uv pulses was 20 ps. The charge was approximately $1.5 \mathrm{nC}$ per macroparticle. For the set of experiments reported in this section, the bunch compressor was turned off.

In a first experiment, the rf-gun phase was varied while keeping the booster-cavity phase on crest. For each rf-gun phase, the macroparticle separation $\Delta \tau_{f}$ was measured at $\mathrm{X} 9$ using the streak camera. The bunch-compression ratio was computed as $\Delta \tau_{f} / \Delta \tau_{\text {cath }}$, where $\Delta \tau_{\text {cath }}$ is the uv-pulse time separation. The results are compared with numerical simulations performed with the program ASTRA [22] in Fig. 4. The measurement of the compression in the rf gun is in good agreement with the numerical model.

In a second set of experiments, the booster-cavity phase was varied while keeping the rf-gun phase at its nominal value of $45^{\circ}$. As before, streak camera measurements at X9 provided the macroparticle time separation for each phase setting. The bunch-compression ratio was inferred by normalizing the macroparticle time separation $\Delta \tau_{f}$ to the time separation when the cavity is operated on crest $\Delta \tau_{f}\left(\psi_{0}=\right.$ 90); as guided by Eq. (6). The latter equation shows no compression; $\mathcal{C}_{b} \simeq 1$ for $\psi_{0} \simeq 90^{\circ}$. The numerical simulations performed with ASTRA and the analytical estimate based on Eq. (6) agree very well with the experimental measurement; see Fig. 5. Unfortunately, we were limited to

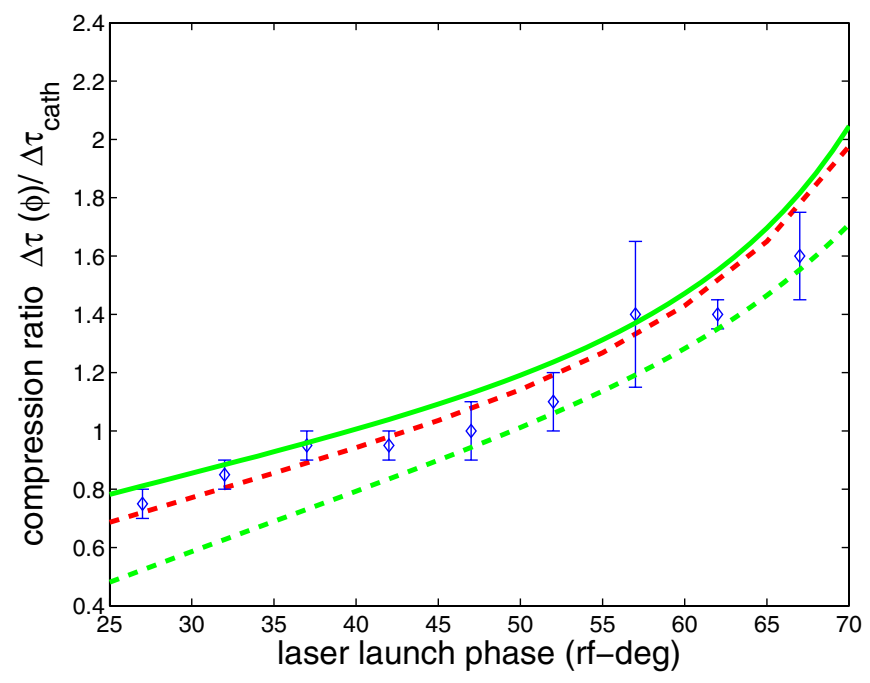

FIG. 4. (Color) Compression factor in the rf-gun cavity for various operating phases. Diamonds are experimental measurements, dashed red, solid green, and dashed green lines correspond, respectively, to numerical simulations with ASTRA, numerical integration of the equation of motion using the field $\hat{E}_{z}$ obtained from SUPERFISH, and numerical integration assuming $\hat{E}_{z}=\cos (k z)$ (following Ref. [16]).

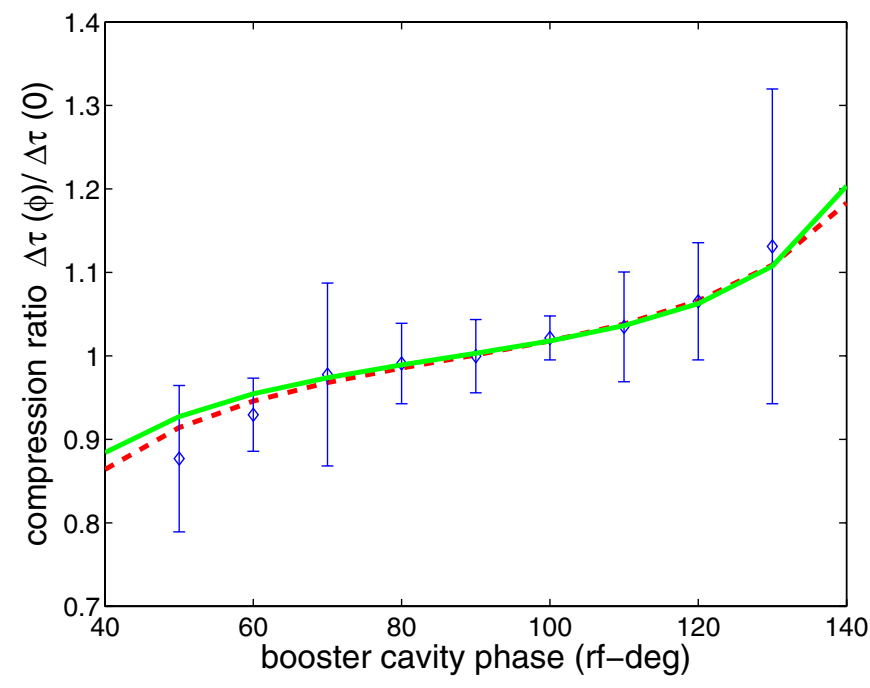

FIG. 5. (Color) Compression factor in the booster cavity for various operating phases. Diamonds are experimental measurements, solid and dashed lines correspond, respectively, to analytical calculation using Eq. (6), and numerical simulations performed with ASTRA . Note: The phase $\psi_{0}=90^{\circ}$ corresponds to maximum acceleration.

phases within $\psi_{0} \in\left[50^{\circ}, 130^{\circ}\right]$, operating at phases outside of this interval resulted in large transverse beam envelope (rf-induced defocusing and chromatic aberrations). This prevented the beam from being transported up to the $\mathrm{X} 9$ viewer without resulting in a poor signal on the streak camera. Hence, we could not measure the onset the simulation predicts for larger off-crest phases.

\section{MAGNETIC BUNCH COMPRESSION}

\section{A. Theoretical background}

In the magnetic-based bunch compressor [23], an energy-dependent path length is introduced via four dipoles arranged to form a dispersion bump henceforth refer to as "chicane." The incoming bunch is first accelerated off crest in the booster cavity such as to introduced a timeenergy correlation along the bunch (the bunch head has a lower energy than the tail). When the bunch passes through the chicane it gets compressed; the tail catches up with the head of the bunch. Under a single-particle approach, using TRANSPORT [24] formalism, an electron with relative longitudinal phase space coordinates $\left(t_{i}, \delta_{i}\right)$ with respect to the bunch center transforms as follows:

$$
t_{f}=t_{i}+\frac{R_{56}}{c} \delta_{i}
$$

where $R_{56}$ is first order momentum compaction associated with the bunch compressor and $c$ is the velocity of light. The fractional energy spread $\delta_{i} \equiv\left(\mathcal{E}_{i}-\langle\mathcal{E}\rangle\right) /\langle\mathcal{E}\rangle$ remains unchanged as long as collective effects are insignificant. The condition for minimum bunch length (i.e. $t_{f}=0$ ) 


$$
\frac{d \delta_{i}}{d t_{i}}=\frac{c}{R_{56}}
$$

provides the required value for the correlation $d \delta_{i} / d t_{i}$ given the bunch compressor $R_{56}$. The value of $R_{56}$ depends solely on the bunch-compressor geometry (bending angle, dipole separations) and was evaluated using numerical simulations. The magnetic fields in each dipole were measured and used in a MATLAB [25] program to integrate the equations of motion using a fourth order Runge-Kutta algorithm [26]. For a nominal bending angle of $22.5^{\circ}$ we present, in Fig. 6, the evolution of beam position and deflection angle in the bunch compressor. Our results agree well with the more refined simulations presented elsewhere [27].

In the case of the two-macroparticle bunch, the evolution of the macroparticle separation $\Delta \tau_{f}$ downstream of the bunch compressor is given by

$$
\Delta \tau_{f}=\Delta \tau_{i}+\frac{R_{56}}{c} \frac{\Delta \mathcal{E}}{\mathcal{E}}+\frac{T_{566}}{c}\left(\frac{\Delta \mathcal{E}}{\mathcal{E}}\right)^{2},
$$

where $\Delta \mathcal{E}$ is the macroparticle energy difference, $\mathcal{E}$ their average energy, and $\Delta \tau_{i}$ their time separation upstream of the compressor. Compared to Eq. (7), Eq. (9) includes a second-order term in $\frac{\Delta \mathcal{E}}{\mathcal{E}}$ to account for the large energy separation between the two macroparticles. The secondorder momentum compaction, $T_{566}$, is related to $R_{56}$ given the chicane geometry: for chicane-type bunch compressors $T_{566} \simeq-\frac{3}{2} R_{56}$ [28]. Hence, from a measurement of the macroparticle time separation and the corresponding energy difference, we can infer the momentum compaction using Eq. (9).
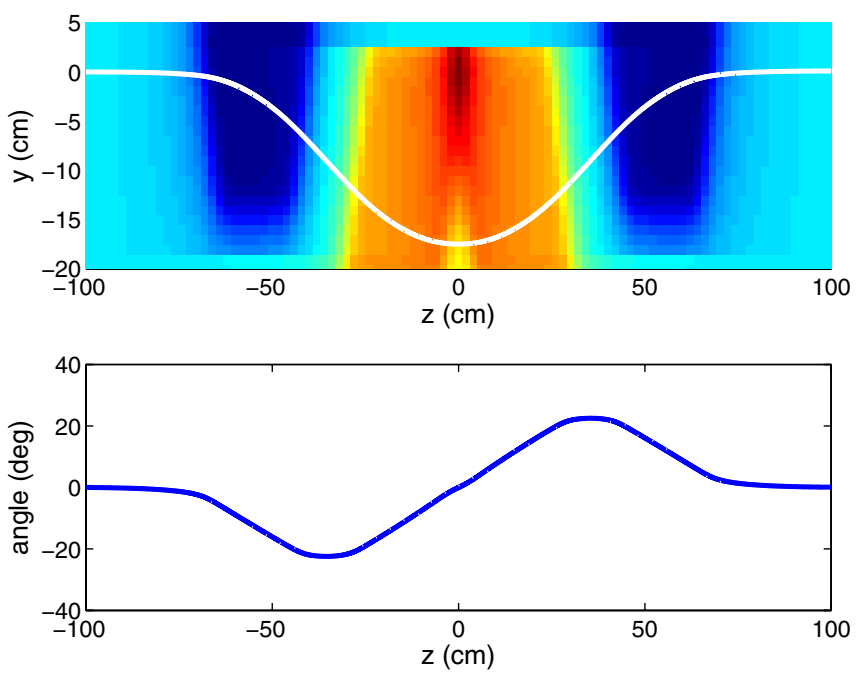

FIG. 6. (Color) Overview of the magnetic bunch-compressor chicane. Top: electron vertical trajectory superimposed to a false color map of the magnetic field $B_{x}(x=0, y, z)$; bottom: electron vertical deflection angle. The electron energy is $12.9 \mathrm{MeV}$, and the maximum B-field of the dipoles are 593.20 and $-592.35 \mathrm{G}$ for, respectively, the outer and inner dipoles.

\section{B. Experimental results and numerical simulations}

An experiment aiming to measure the momentum compaction of the bunch compressor was performed. The beam energy was varied by changing the operating phase of the booster cavity while the bunch-compressor dipole currents were kept constant. Thus, as the energy is varied, the bending angle and thereby the chicane $R_{56}$ is varied. The rf gun was operated at the nominal values reported in Table I. For each beam-energy setting, the initial macroparticle time separation $\Delta \tau_{i}$ and the corresponding energy separations (see example in Fig. 7) were measured with the bunch compressor turned off. The bunch-compressor dipoles were then set to their nominal values and the macroparticle time separations downstream of the compressor $\Delta \tau_{f}$ was measured. For the setting corresponding to maximum compression $(\mathcal{E}=12.9 \mathrm{MeV})$, the phase of the booster cavity was tuned so as to cause the two macroparticles to overlap in time. Apart from the maximum-compression case $\left(-35^{\circ}\right)$, we used the phase settings of $\pm 20^{\circ}$ off crest. For the latter cases, the change in time of macroparticle separation is only a factor of 3 bigger than the streak camera instrumental error. The values of $R_{56}$, directly estimated from Eq. (9), are gathered in Table II along with simulation results. In general the agreement between simulation and experiment is good. The measurement, however, suffers from large error bars away from the maximum-compression phase. These large error bars are mainly due to the streak camera resolution ( $2 \mathrm{ps})$, and could be significantly reduced by using state-of-art streak cameras with $\sim 200$ fs resolution.
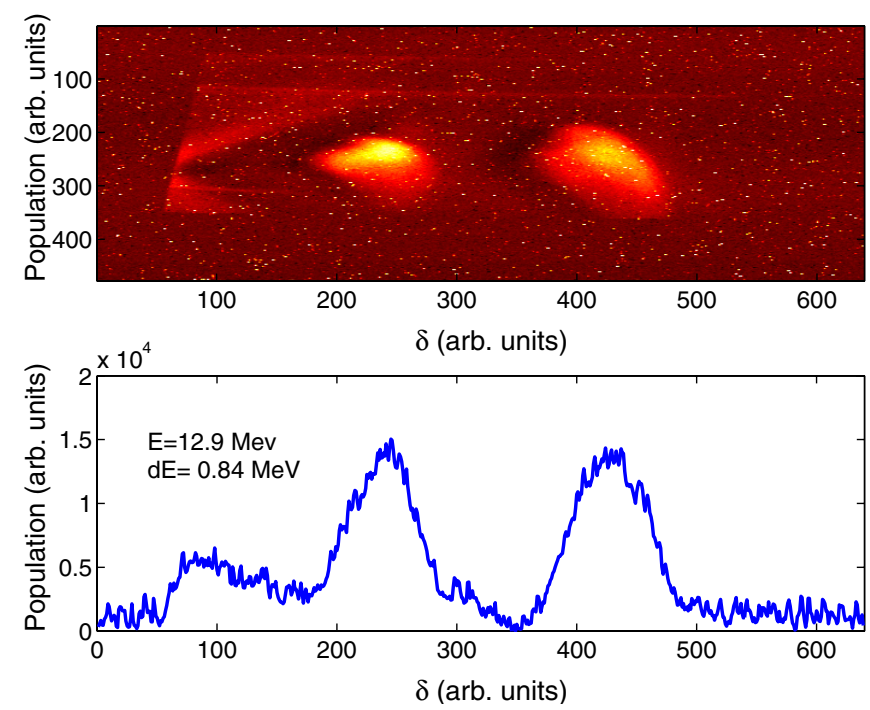

FIG. 7. (Color) Example of energy distribution of the twomacroparticle bunch. The top figure is the beam transverse density recorded on a screen downstream of the spectrometer (horizontal axis is dispersive axis) and the bottom plot is the corresponding fractional momentum distribution. The bunch mean energy is $\mathcal{E}=12.9 \mathrm{MeV}$ for the data presented. 
TABLE II. Measured versus simulated $R_{56}$. The chicane dipoles current was kept constant [to $1.8 \mathrm{~A}$ (for the outer dipoles)], the bending angle (and thus $R_{56}$ ) are a function of the energy of the incoming two-macroparticle bunch. The case of maximum compression (referred to as max. comp.) corresponds to the nominal bending angle of $22.5^{\circ}$.

\begin{tabular}{lcc}
\hline \hline & $\left|R_{56}\right|(\mathrm{cm})$ & $\left|R_{56}\right|(\mathrm{cm})$ \\
Energy (MeV) & Measured & Simulated \\
\hline 14.5 & $8.3 \pm 3.7$ & 7.47 \\
14.3 & $7.7 \pm 2.8$ & 7.82 \\
12.9 (max. comp.) & $9.1 \pm 1.1$ & 8.89 \\
\hline \hline
\end{tabular}

\section{CONCLUSIONS}

We have developed and implemented a method, based on the generation of a two-macroparticle bunch, to characterize the longitudinal single-particle beam dynamics. This implementation is straightforward in linacs incorporating a photoemission electron source. We presented its application in a photoinjector to measure directly the compression factor of a rf gun and an accelerating cavity. The method also allows direct measurement of the momentum compaction of a magnetic bunch compressor. The twomacroparticle bunch method could have promising applications in commissioning of linacs with complex longitudinal phase space manipulation (e.g. multistage compression such as in the vacuum ultraviolet free-electron laser in DESY [29]) to verify the longitudinal dynamics is performing per design. Other potential applications include the exploration of collective effects such as wake field or coherent synchrotron radiation (CSR) [30]. In these latter applications a measurement of delayed-macroparticle energy versus the delay time between the two macroparticles could yield information on the wake potential (or "overtake" potential for CSR). Finally, the technique can be in principle extended to probe nonlinear effects in the longitudinal single-particle beam dynamics by generating a multimacroparticle bunch.

\section{ACKNOWLEDGMENTS}

We wish to acknowledge C. L. Bohn of Northern Illinois University, H. Edwards of Fermilab, and A. Melissinos of University of Rochester for comments and fruitful discussions. We are thankful to J. Li of University of Rochester for calibrating the streak camera. This work was supported by Universities Research Association Inc. under Contract No. DE-AC02-76CH00300 with the U.S. Department of Energy and by the Northern Illinois Center of Accelerator and Detector Development (NICADD).

[1] See, for instance, LCLS Conceptual Design Report, SLAC-R-593/UC-414, 2002; TESLA Technical Design
Report Imprint No. DESY 2001-011 or ECFA 2001-209, 2001.

[2] N. Barov et al., Phys. Rev. ST Accel. Beams 3, 011301 (2000).

[3] G. A. Krafft, in Proceedings of the Microbunches Workshop, AIP Conf. Proc. No. 367 (AIP, New York, 1996), pp. 46-55.

[4] G. A. Krafft, in Proceedings of the 1997 IEEE Particle Accelerator Conference (PAC 97), Vancouver, B.C., Canada (IEEE, Piscataway, NJ, 1997), pp. 2268-2270.

[5] P. Piot, D. R. Douglas, and G. A. Krafft, Phys. Rev. ST Accel. Beams 6, 030702 (2003).

[6] G. A. Krafft, B. A. Bowling, M.T. Crofford, and J.C. Hovater, Nucl. Instrum. Methods Phys. Res., Sect. A 557, 314 (2006).

[7] D. Mihalcea et al. (unpublished).

[8] For information see http://nicadd.niu.edu/fnpl.

[9] B. Aune et al., Phys. Rev. ST Accel. Beams 3, 092001 (2000).

[10] M.J. Fitch et al., in Proceedings of the 20th Linac Conference, Monterrey, CA, 2000, Paper No. MOB16, also available on physics/0008206.

[11] The streak camera model is Hamamatsu C5680-21S.

[12] D. Mihalcea et al., in Proceedings of 2005 IEEE Particle Accelerator Conference (PAC 05), Knoxville, TN (IEEE, Piscataway, NJ, 2005), pp. 4254-4256.

[13] U. Happek et al., Phys. Rev. Lett. 67, 2962 (1991).

[14] M. J.E. Golay, Rev. Sci. Instrum. 18, 347 (1947).

[15] H. Haimson, Nucl. Instrum. Methods 39, 13 (1966).

[16] K.-J. Kim, Nucl. Instrum. Methods Phys. Res., Sect. A 275, 201 (1989).

[17] B.E. Carlsten and D.C. Nguyen, Part. Accel. 56, 127 (1997).

[18] L. Serafini and M. Ferrario, in Physics of, and Science with, the X-ray Free-Electron Laser edited by S. Chattopadyay et al., AIP Conf. Proc. 581 (AIP, New York, 2001), pp. 87-106.

[19] P. Piot, L. Carr, W. S. Graves, and H. Loos, Phys. Rev. ST Accel. Beams 6, 033503 (2003).

[20] S. Anderson et al., Phys. Rev. ST Accel. Beams 8, 014401 (2005).

[21] Los Alamos Accelerator Code Group, Poisson/Superfish Reference Manual, LA-UR-87-126, 1987.

[22] K. Flöttmann, ASTRA user manual, DESY, 2000 (information available on the web site http://www.desy.de/ $\sim$ mpyflo).

[23] J. B. Rosenzweig, N. Barov, and E. Colby, IEEE Trans. Plasma Sci. 24, 409 (1996).

[24] K. L. Brown, D. C. Carey, Ch. Iselin, and F. Rothacker, CERN 73-16 (revised as CERN 80-4), CERN, 1980.

[25] MATLAB is available from MathWorks, Inc.

[26] W. H. Press, B.P. Flannery, S. A. Teukolsky, and W. T. Vetterling, Numerical Recipe in C: The Art of Scientific Computing (Cambridge University Press, Cambridge, England, 1992), 2nd ed.

[27] J.-P. Carneiro et al. in Proceedings of the Eighth European Particle Accelerator Conference (EPAC2002), Paris, edited by T. Garvey, J. Le Duff, P. Le Roux, C. PetitJean-Genaz, J. Poole, and L. Rivkin (EPS-IGA, Geneva, 2002), pp. 759-761.

[28] T. O. Raubenheimer, P. Emma, and S. Kheifets, in 
Proceedings of the 1993 Particle Accelerator Conference, (PAC93), Washington, D.C. (IEEE, Piscataway, NJ, 1993), IEEE catalogue 93CH3279-7, pp. 635-637.

[29] T. Limberg, Ph. Piot, and F. Stulle, in Proceedings of the Eighth European Particle Accelerator Conference
(EPAC2002), Paris, edited by T. Garvey, J. Le Duff, P. Le Roux, C. Petit-Jean-Genaz, J. Poole, and L. Rivkin (EPS-IGA, Geneva, 2002), pp. 811-813.

[30] See, for instance, C. L. Bohn, AIP Conf. Proc. 647, 81 (2002). 